\title{
DRAFT: MULTI-OBJECTIVE OPTIMIZATION IN AXIAL COMPRESSOR DESIGN USING A LINKED CFD-SOLVER
}

\author{
Kai Becker; Martin Lawerenz \\ Department of Turbomachinery \\ Institute of Thermal Engineering \\ University of Kassel \\ D-34109 Kassel \\ Email: kai.becker@uni-kassel.de
}

\author{
Christian Voss, Reinhard Moenig \\ German Aerospace Centre \\ Institute of Propulsion Technology \\ Linder Hoehe \\ D-51147 Cologne \\ Email: christian.voss@dlr.de
}

\begin{abstract}
In combination with a multi-objective $3 D$ optimization strategy, a linked CFD-solver is introduced in this paper, combining 3D-Reynolds-averaged-Navier-Stokes and an inviscid throughflow method. It enables the adjustment of the $3 D$ boundary conditions for any design variation and contains new options for configuring the objective functions. The linking is achieved by matching the flow information between both CFD codes in an iterative procedure. Compared to an individual $3 D$-CFD calculation, the convergence does not take significantly longer.

The potential of the linked CFD-solver is demonstrated in a multi-objective optimization for one blade row to be optimized and one operating point at a 3-stage axial compressor with inlet guide vane. In the optimization, the objective functions are formulated, so that the performance of the axial compressor is enhanced in addition to the improved efficiency in the $3 D$-cascade.
\end{abstract}

\section{INTRODUCTION}

Today's turbomachinery design process requires the intensified application of CFD technologies in combination with optimization strategies due to constricted research and development budgets. In design optimization, evolutionary and genetic algorithms are used more frequently because of their robustness related to data's noise, their capability of feasible regions and their massively parallelized characteristics [1]. This stochastic optimization algorithm, however, requires a large number of CFD calculations so that diverse speedup techniques are applied to decrease the set of necessary evaluations like neural networks [2,3] or kriging approximation models [4].

Current computing powers and sophisticated 3D flow solvers enable the use of optimization tools in the multistage axial compressor design to amend the blade performance of single stages or blades three dimensionally. The optimization of an entire multistage compressor surpasses present standards. In this paper, the problem is evaded by the use of a linked flow solver combining the 3D flow solver with an inviscid through flow method modeling losses and deviation. The 2D simulation is used to raise the reliability of the optimization process by updating the boundary conditions for each design and illustrating the influence on the whole engine.

The paper is subdivided into three parts. Initially, the principle of the linked solver is described in detail. In the second section the multi-objective optimization package, developed over the past years at the German Aerospace Centre in Cologne [5], is introduced briefly focusing on the linked process chain. The paper is concluded with the potential application of the linked CFD-solver using the example of the axial compressor IDAC3 of RWTH Aachen. For the time being, the optimization is performed for the blades of the third rotor and one operating point, the design point. In addition to the improvements of the flow field by the three dimensionally designed rotor blades, the performance of the entire axial compressor is enhanced, leading to the conclusion. 


$\begin{aligned} & \text { NOMENCLATURE } \\ & \text { Latin }\end{aligned}$
$\begin{array}{ll}\mathrm{DF} & \text { diffusion factor } \\ \mathrm{m} & \text { mass flow rate } \\ \mathrm{Ma} & \text { Mach number } \\ \mathrm{r} & \text { radius } \\ \mathrm{s} & \text { entropy }\end{array}$

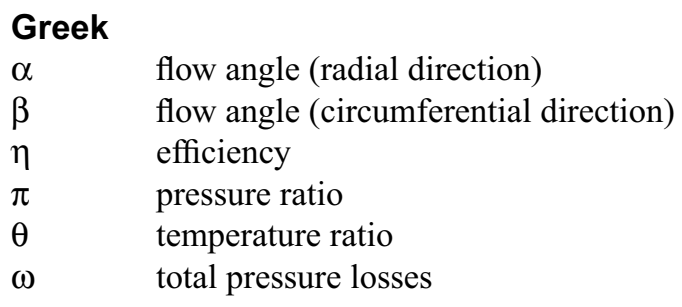

\section{Abbreviations}

RANS Reynolds-averaged Navier-Stokes

Ref reference (initial design)

Rot3 third rotor of IDAC3

Sta2 second stator of IDAC3

Sta3 third stator of IDAC3

OP1 operating point (design point)

Opti2 second optimal design

Opti3 third optimal design

\section{Indices}

0 inlet

1 leading edge

2 trailing edge

3 outlet

is isentropic

$\mathrm{m} \quad$ meridional

pol polytropic

$\mathrm{r}$ radial

rel relative

stat static

$\mathrm{t}$ total

u circumferential

\section{LINKED SOLVER}

The here presented linked solver consists of a meridional throughflow model and a 3D-simulation. As throughflow model, the in-house program MAGELAN is used. Based on a pressure correction method, correlations are included modeling losses and deviation (see [6]). The 3D simulation is done by the DLR-code TRACE. The implicit Reynolds-averaged Navier-Stokes solver uses a cell-centered finite volume scheme and a two equation $\mathrm{k}-\omega$ model for turbulence closure (see $[7,8]$ ).

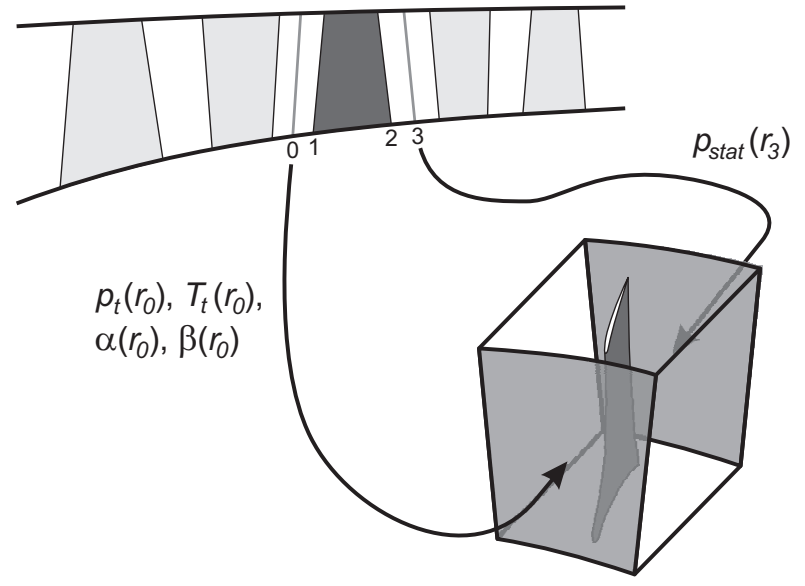

Figure 1. EXCHANGE OF FLOW INFORMATION I: DEFINING THE BOUNDARY CONDITIONS FOR 3D-RANS

The main issue is the linkage of the boundary conditions and blade characteristics between both CFD-codes. In this context, the entry and exit plane of the 3D-RANS have to be part of the throughflow grid to enable simple exchange of flow data. For the 3D-RANS simulation, the entry and exit boundary conditions are extracted from the throughflow model as shown in Fig. 1. At the entry, these are the distributions of total pressure $p_{t}$, total temperature $T_{t}$, and the two flow angles in circumferential direction $\alpha$ and radial direction $\beta$ as well as the static pressure distribution $p$ at the exit.

Within the throughflow model, correlations are required to model losses and deviation of rotor and stator rows and they are outlined later on. For the blade rows, being part of the three dimensional simulation, the results of the correlations have to be replaced. At the trailing edge, velocity and total pressure are circumferentially averaged as illustrated in Fig. 2. Hence, the radial distributions of the loss coefficient and the flow angle at the trailing edge can be determined in return.

The process starts with a 2D throughflow simulation because this is far less time- and cost-consuming in contrast to the 3DRANS calculation. For all blades, the internal 2D correlations are used. If the 2D simulation is successful, the boundary conditions for the 3D-RANS are specified and sent to the 3D code which is started subsequently.

After a specified number of iterations, the simulation is halted, and the radial distributions of loss coefficient and flow angle are calculated for another 2D simulation. In the following throughflow calculations, this information is used instead of the internal correlations. The 3D boundary conditions are updated with the recent results and the 3D-RANS is resumed. This sequence is repeated until the variations of flow characteristics are negligible and the 3D-RANS is converged. Figure 3 shows that three repetitions of the loop are sufficient to achieve a converged 


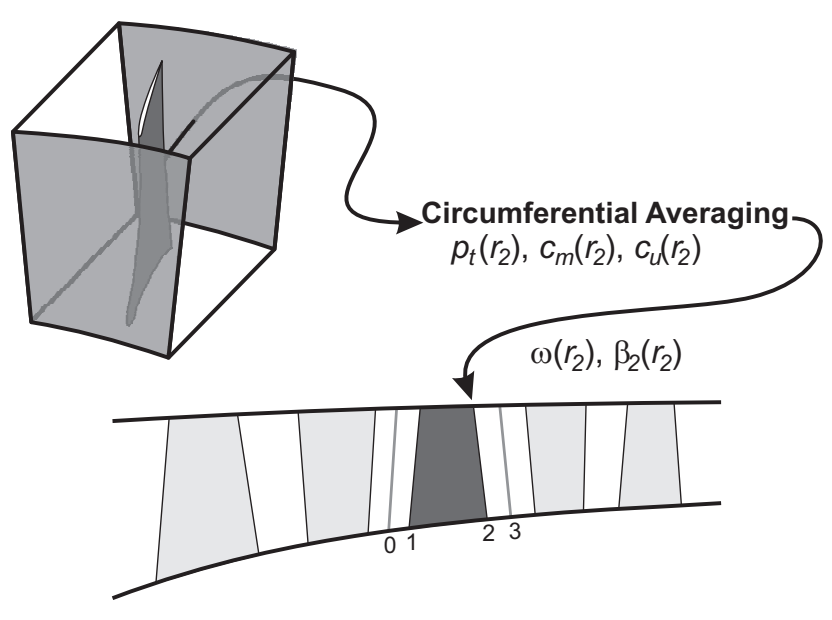

Figure 2. EXCHANGE OF FLOW INFORMATION II: UPDATING RADIAL DISTRIBUTIONS OF LOSS COEFFICIENT AND OUTLET FLOW ANGLE

solution.

\section{Correlations for Losses and Deviation}

In order to take into account viscous effects, correlations for the loss of total pressure and models to predict the difference between flow angles and corresponding geometric angles of the airfoils are included. These correlations are derived from [9] and [10]. Depending on geometric data and aerodynamic loading, losses are described by the total pressure loss coefficient. The overall loss is composed of design and off design losses due to incidences. Profile and end wall boundary layers are included, as well as shocks, secondary flow and tip clearance effects. Finally, values of the total pressure loss coefficient are corrected to take the actual Reynolds number into account. Within the throughflow method a viscous body force acting in opposite flow direction is used to produce the predicted losses.

\section{Convergence}

The convergence of the linked solver is complex, because the throughflow simulation and the $3 \mathrm{D}$ simulation have to converge both separately and conjointly. The convergence criteria for the 3D simulation are the mean residual, the isentropic efficiency and the mass flow difference between inlet and outlet plane. The magnitude of pressure corrections is usually used within the numerical algorithm of the throughflow method to assess the rate of convergence. But, leadoff tests have shown that the variations of total pressure ratio and polytropic efficiency throughout the last iterations are better suited. The calculation is considered as converged if the variations fall below a certain threshold.

The overall convergence is illustrated in Fig. 3 for the 3rd

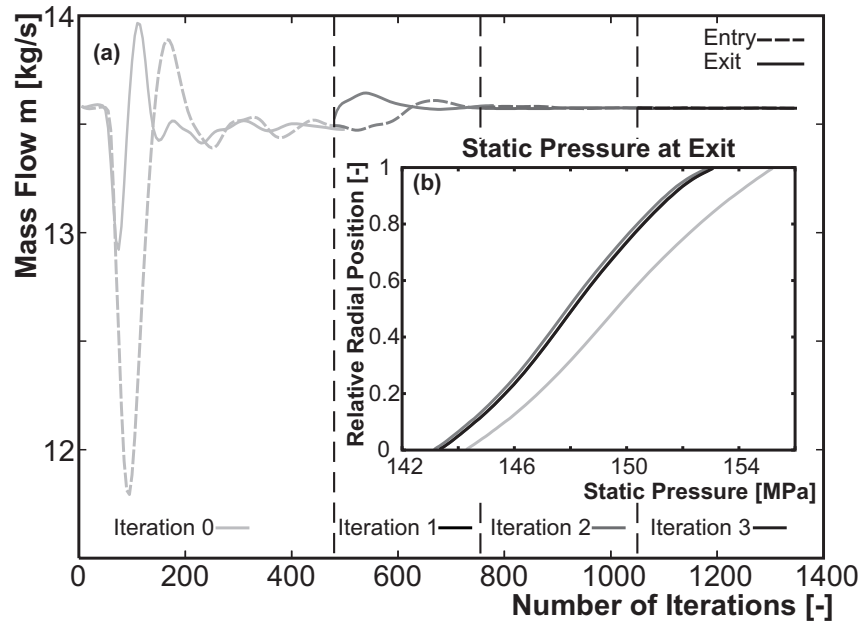

Figure 3. CONVERGENCE OF THE LINKED SOLVER: (a) DEVELOPMENT OF MASS FLOW AT ENTRY AND EXIT OF 3D-RANS SIMULATION; (b) 3D EXIT BOUNDARY CONDITION FOR EACH ITERATION, RADIAL DISTRIBUTION OF STATIC PRESSURE

rotor of IDAC3 in the design point. Following the criterion of the $3 \mathrm{D}$ case, the mass flow at inlet and outlet plane is used. Figure $3 \mathrm{a}$ is showing the progress of both mass flows. The dashed lines are marking updates of the $3 \mathrm{D}$ boundary conditions by $2 \mathrm{D}$ throughflow simulations. As mentioned before, the process starts with boundary conditions extracted from an initial 2D simulation. The trend of the inlet mass flow is very smooth and thus not influenced significantly by updates of the 3D boundary conditions. The progression of the outlet mass flow is showing steps when updating the boundaries. In Fig. 3b, the 3D exit boundary condition, the radial distribution of the static pressure, is being depicted for each iteration. The large gap between iteration 1 and iteration 2 is explained by the use of the 2D correlations in the initial throughflow calculation. The combined 3D simulation converges after approximately 1300 iterations. Therefore, the linking does not slow down the process chain in contrast to a sole TRACE calculation.

\section{Limits of Flow Angle and Loss Coefficient in the Throughflow Calculation}

Due to the different characteristics of the two CFD codes, the circumferentially averaged radial distribution of flow angle and loss coefficient cannot be transferred directly. Consequently, the information extracted from the 3D-RANS calculation has to be modified for the use in the throughflow model. For the flow angle at the trailing edge, the gradient has to be limited because secondary flows lead to large deflections in the endwall boundary layers within the RANS solution. Whereas shear stresses in these viscous areas are neglected in the throughflow method. In Fig. 4, the alteration of the flow angle is shown in contrast to the original distribution. They differ particularly in the blade tip region. 


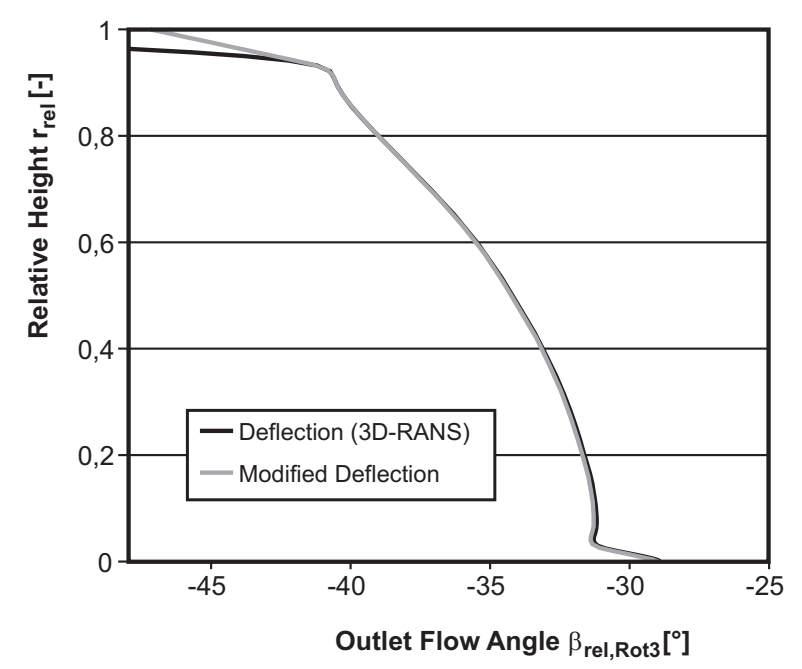

Figure 4. MODIFIED DEFLECTION FOR USE IN THE THROUGHFLOW MODEL

Shear stresses in the endwall zones may lead to very high local loss coefficients when extracted from the 3D simulation. This could produce unreasonable flow situations within the throughflow method because endwall boundary layers are not resolved. Hence, the losses have been limited by a maximum total pressure loss coefficient of $40 \%$ for the present calculations.

\section{MULTI-OBJECTIVE OPTIMIZATION STRATEGY}

An asynchronous multi-objective evolutionary algorithm is applied as optimization strategy, accelerated additionally by various response surface models. The optimization code is parallelized with MPI and consists of a controlling root process and a number of slave processes. In the root process, the database of all evaluated members is governed according to the Pareto optimality [11] and new members are derived using mutation, crossover and differential evolution as well as the additional response surfaces.

They are trained separately due to the basic modular concept and lower required computing power. Their training is performed frequently in the running process, based on the present database. The new detected auspicious candidates then are sent to the optimization and calculated subsequently by the linked CFD-solver. Thereby, neural networks, Kriging procedures and polynomial response surfaces are utilized.

In the slave processes the members are evaluated making use of a process chain. The details of the entire optimization algorithm are presented in $[5,12]$. Consequently, only modifications with respect to the linked solver are described here.

According to Fig. 5, the process chain can be subdivided into four parts, the alteration of the geometry, the generation of the S1- ,S2- and 3D-grids, the flow simulation and the postprocessing.

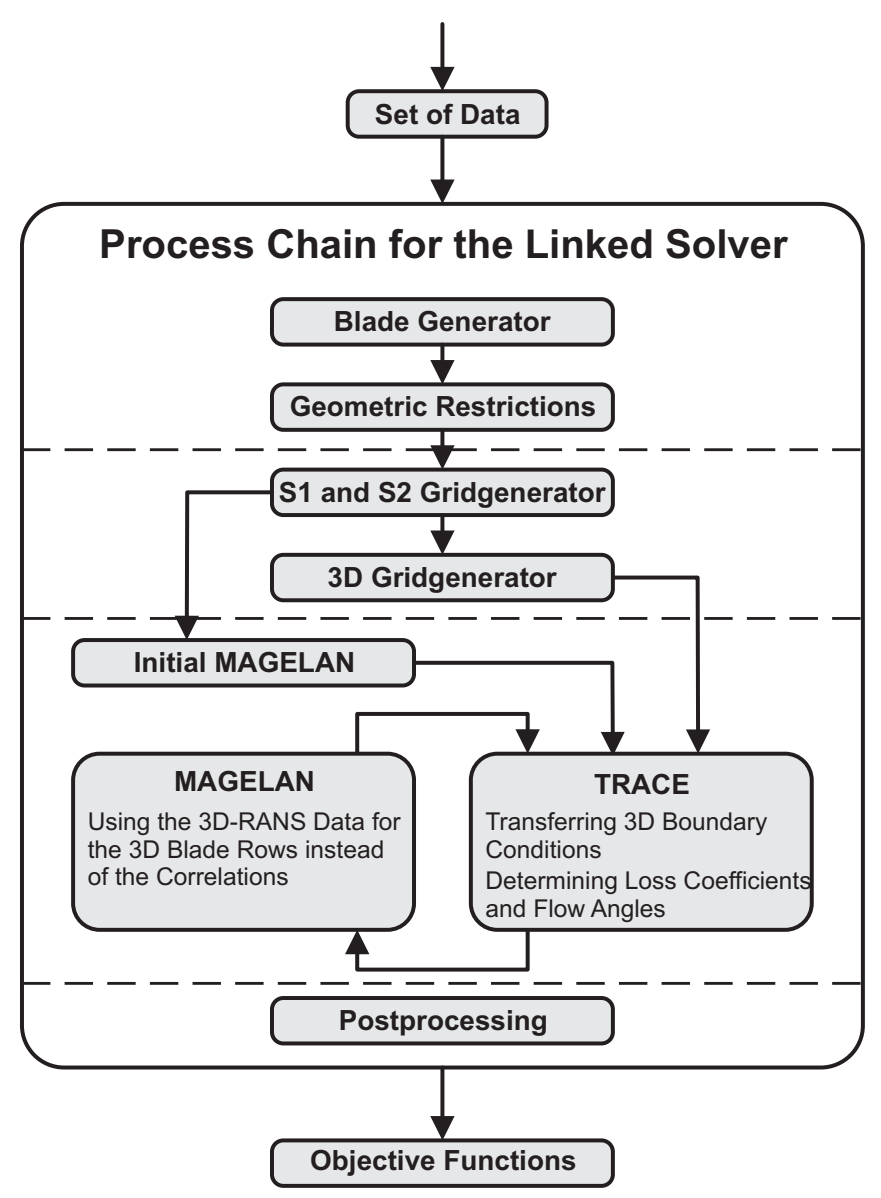

Figure 5. PROCESS CHAIN FOR THE LINKED SOLVER

\section{Alteration of Geometry}

The slaves receive the set of data (member) from the optimization process in a normalized form. To meet the standards of the Blade Generator the variables have to be rescaled into real values. The Blade Generator is a CAD tool developed at the institute of propulsion technology of DLR based on B-spline curves and B-spline tensor product surfaces (see [13]). Its wide design freedom may lead to unfeasible blade shapes according to mechanical and structural aspects. So geometric restrictions have to be applied to prevent the transmission of impossible geometric designs. The applied geometric restrictions are an enhanced version of the ones used in 2D studies described in [5]. First, $2 \mathrm{D}$ restrictions for each profile are investigated. Then the whole 3D airfoil is checked by an extremum and a monotony criterion for the stagger line, geometric inlet and outlet blade angle, radius and thickness. The process chain is being abandoned, if the design fails the geometric restrictions in any part. In these cases, the member gets a bad artificial objective value and is sent back to the root process. 


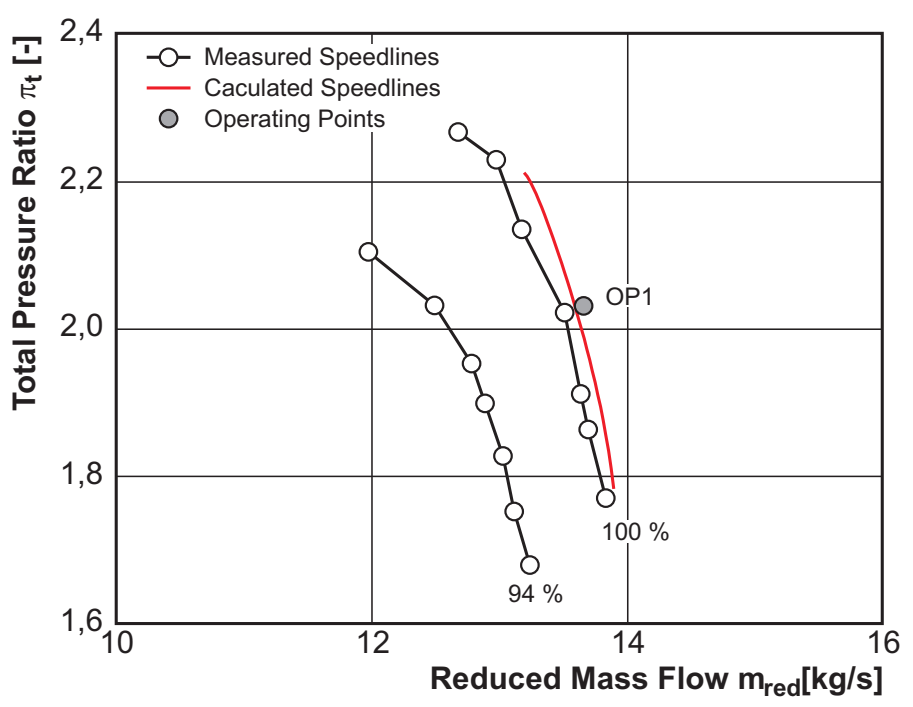

Figure 6. COMPRESSOR MAP OF IDAC3

\section{Grid Generation}

The grid generation is implemented in two parts. The S1 and S2 grids are generated by an extension of CFD Norway's G3DMESH [14]. For the linked solver, the throughflow grid is created and is matched with the S2 grid so that entry and exit plane of the 3D computational grid are grid lines of the throughflow method. The 3D multi-block grid also is built by G3DMESH. This procedure uses the existing S1 grids of the hub and the tip surface and the S2 grid to merge the complete 3D mesh automatically. If the grid generation fails, the process chain is being abandoned and the member is being badly benchmarked.

\section{Linked Solver}

The initial throughflow calculation is started using the new grid but the original information for the correlations. Furthermore, the simulation is executed as previously described. A failure in one iteration stops the process chain and degrades the objective functions.

\section{Postprocessing}

After the linked solver has been successfully converged, the postprocessing of both CFD tools is done and the flow parameters are determined so that the objective functions can be calculated and returned to the optimization loop. Hence, each member receives a certain quality rating, and they all are collected and evaluated in a global database for further treatment by the optimizer.

A special feature of the optimization tool is the recording of flow parameters for each member besides the objective values and the normalized design. Therefore, certain members in the global database can be reproduced or recalculated for a deeper

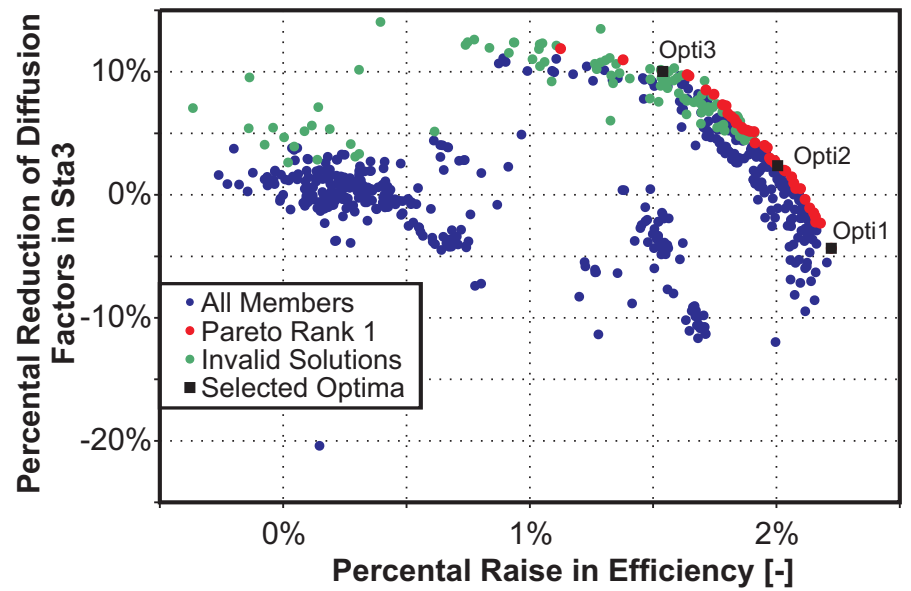

Figure 7. OBJECTIVES DIAGRAM DISPLAYING THE PERCENTAL VARIATION RELATED TO THE INITIAL DESIGN

insight at any time. Additionally, the composition of the objective functions can be changed without losing the knowledge of the already determined members. Thus, the objective functions can be rearranged if the early formulation misleads. The members' objective functions only have to be reevaluated and rerated in the database. Then, the optimization can proceed.

\section{OPTIMIZATION OF IDAC3'S THIRD ROTOR}

As a test case, the compressor IDAC3 has been selected for the presented first aerodynamic investigation and optimization in order to rely on well-known measurement and verification. The compressor is built with Controlled Diffusion Airfoils. Detailed information of this compressor is given in [15-17].

In Fig. 6, IDAC3's compressor map is illustrated. The experimental data is taken from [16]. In addition, the design point measured in [15] is plotted as OP1. MAGELAN's results for the $100 \%$ speed line also are illustrated. Its speed line is close to the measurements indicating that the used correlations sufficiently represent losses and deviations.

The main purpose of this optimization is the verification and validation of the linked solver associated with the multi-objective optimization tool. Therefore, one operating point and the rotor of the third (and last) stage are used for optimization owing to the required computing time. The blade row is selected for an analysis of the optimizing design process, featuring neighbors on either side.

In total 23 variables have been chosen as design parameters concentrated all on rotor 3 . The $3 \mathrm{D}$ blade design is composed of five construction profiles positioned at hub, midspan and tip as well as at $20 \%$ and $80 \%$ relative blade height. The profile at hub is fixed, whereas a limited shift of the profiles, orthogonally to the direction of the chord length, is allowed for the other four. 

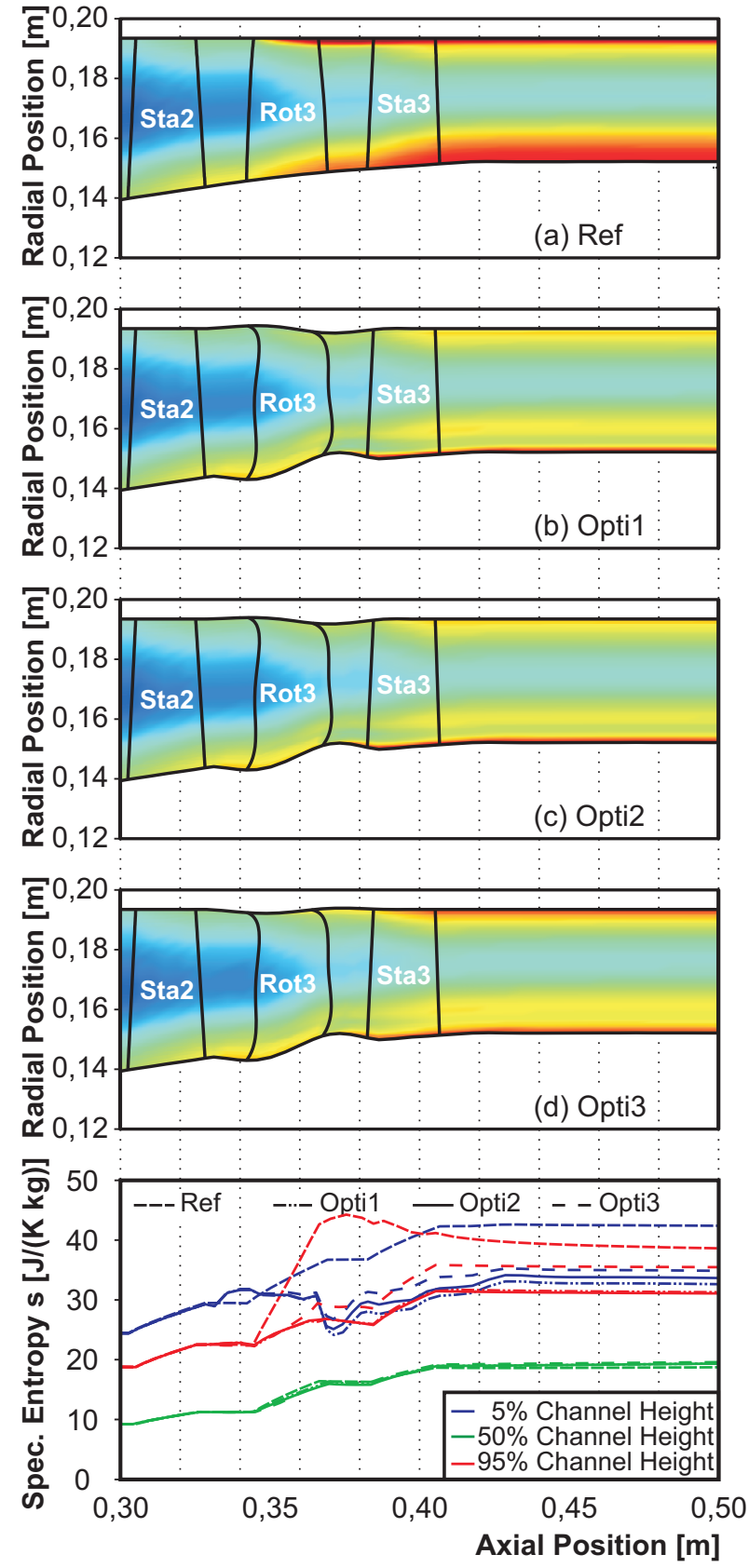

Figure 8. S2 ENTROPY DIAGRAM OF IDAC3'S LAST STAGE FOR THE OPTIMIZED DESIGNS AND THE REFERENCE

Additionally, for all five profiles the stagger line can vary and one control point on the suction side can be moved in axial and radial direction. Furthermore, the shape of hub and tip can be changed by two points being relocatable in radial direction.

In the optimization process, slightly more than 1000 members have been evaluated. Two objectives have been defined. The
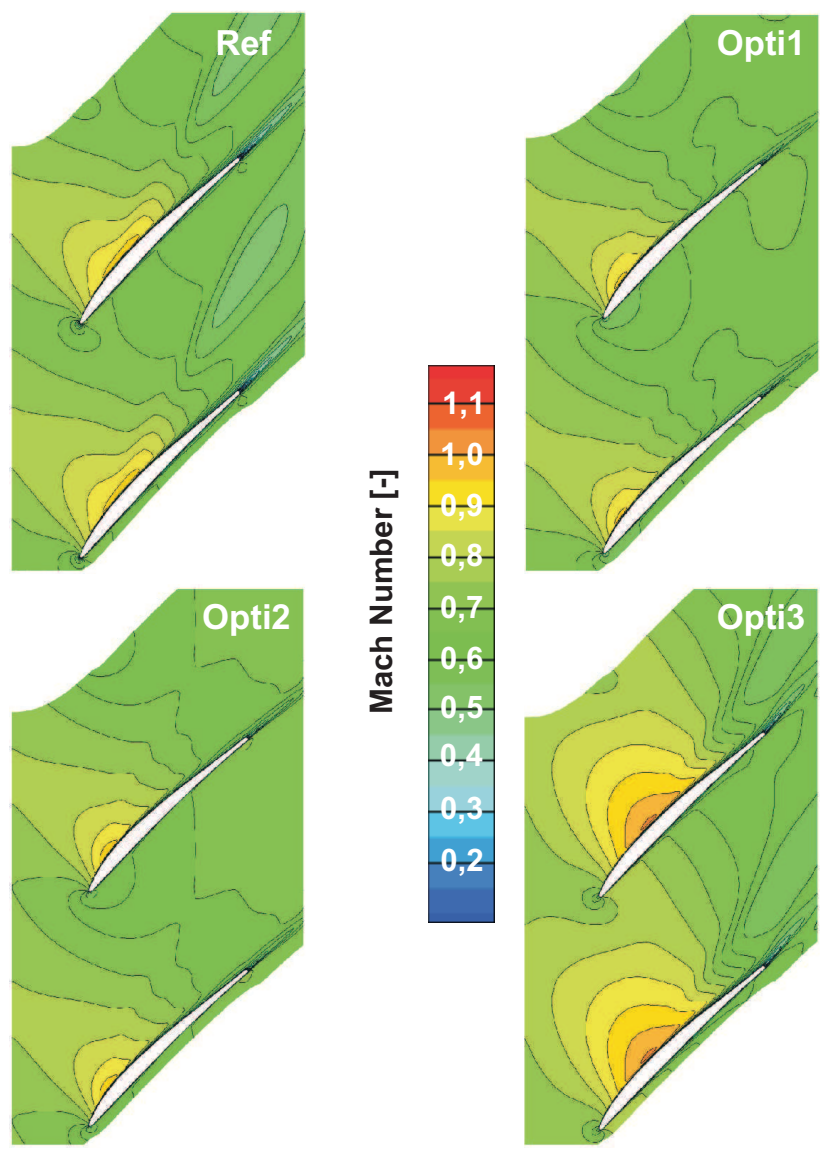

Figure 9. DISTRIBUTION OF MACH NUMBER IN S1 SECTION AT 90\% HEIGHT

goal of the first objective is the improvement of the efficiency. It is composed of the sum of the efficiency of rotor 3, only using the 3D flow parameters of TRACE, and the efficiency of the entire axial compressor, applying MAGELAN's flow data. Whereas, the second objective demands a reduction in the distribution of the diffusion factors in stator 3 . Hence, the maximum and the averaged diffusion factor are summed up here. As an additional restriction, the overall total pressure ratio is not allowed to drop below $\pi_{t}=2$ so that the operating point is retained.

In Fig. 7, the development of the Pareto front is illustrated. With reference to the initial design, the percental reduction of the diffusion factors, the second objective, is plotted versus the percental gain in efficiency (first objective). The optimization proceeds towards the right upper corner. The first members of the optimization are located around the initial design. The larger steps in the developing of the Pareto front show the accelerating effect by using response surface methods. In this test case, the kriging method is applied. The spreading of the members corresponds to a developed Pareto front. The green dots symbolize members that are converged, but feature an undersize overall 

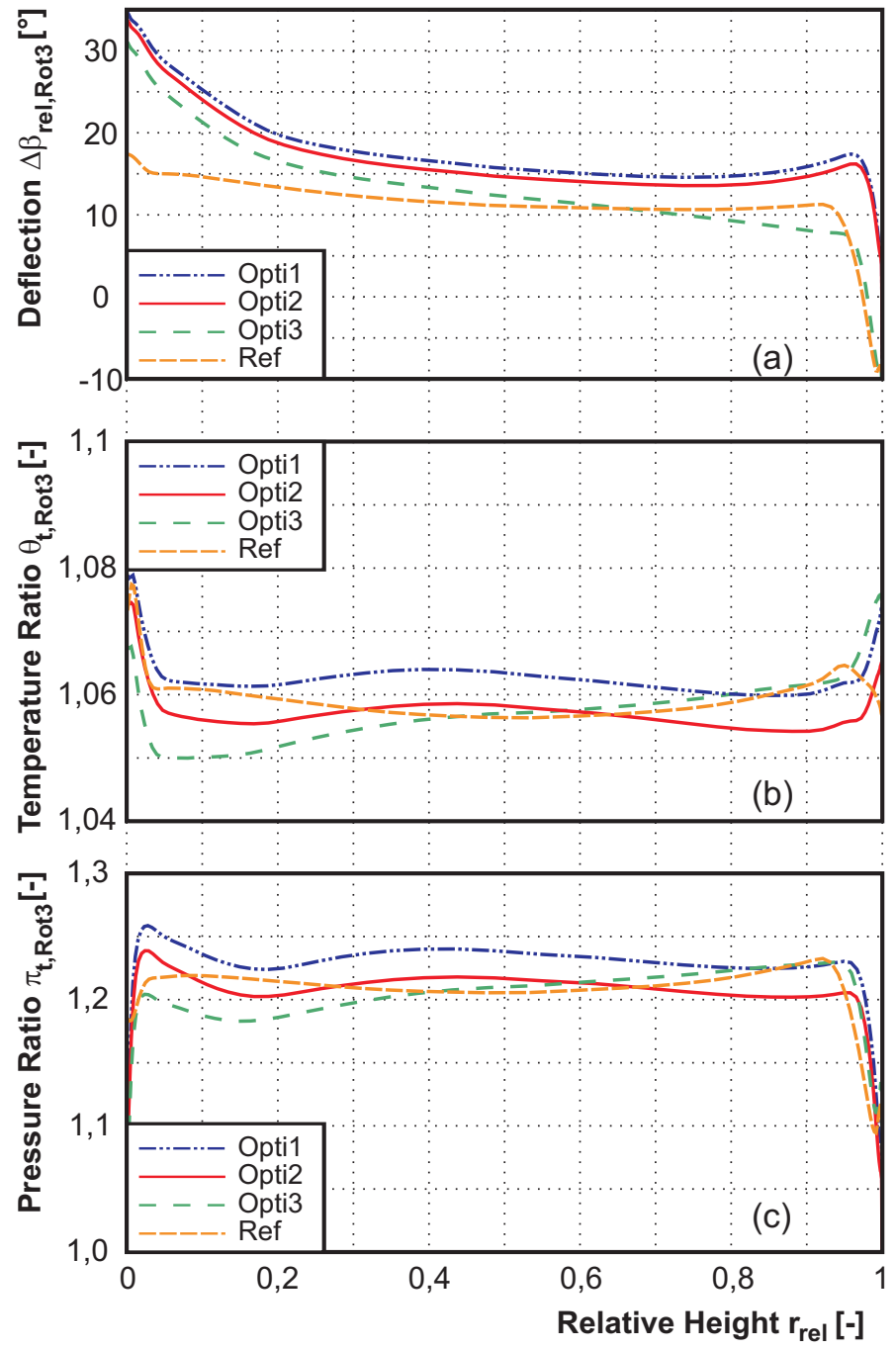

Figure 10. COMPARISON OF NORMALIZED RADIAL DISTRIBUTIONS BETWEEN THE OPTIMIZED DESIGN AND THE ORIGINAL DESIGN AS REFERENCE FOR : (a) DEFLECTION; (b) TOTAL TEMPERATURE RATIO; (c) TOTAL PRESSURE RATIO

total pressure ratio. For the further analysis, three members of Pareto rank 1, marked with a black square, are compared to the original design.

Opti1: emphasis on efficiency

Opti2: compromise between both objectives

Opti3: emphasis on the diffusion factors

In Fig. 8 and Fig. 12, their design differences are shown. Figure 8 shows that the flow path is expanded at the entry of rotor 3 and contracted at the exit for all three. In doing so, the two shiftable points lying on the hub curve impinge upon their limiting values, the first one upon the lower limit and the second one upon the upper limit, respectively. The tip curve varies among
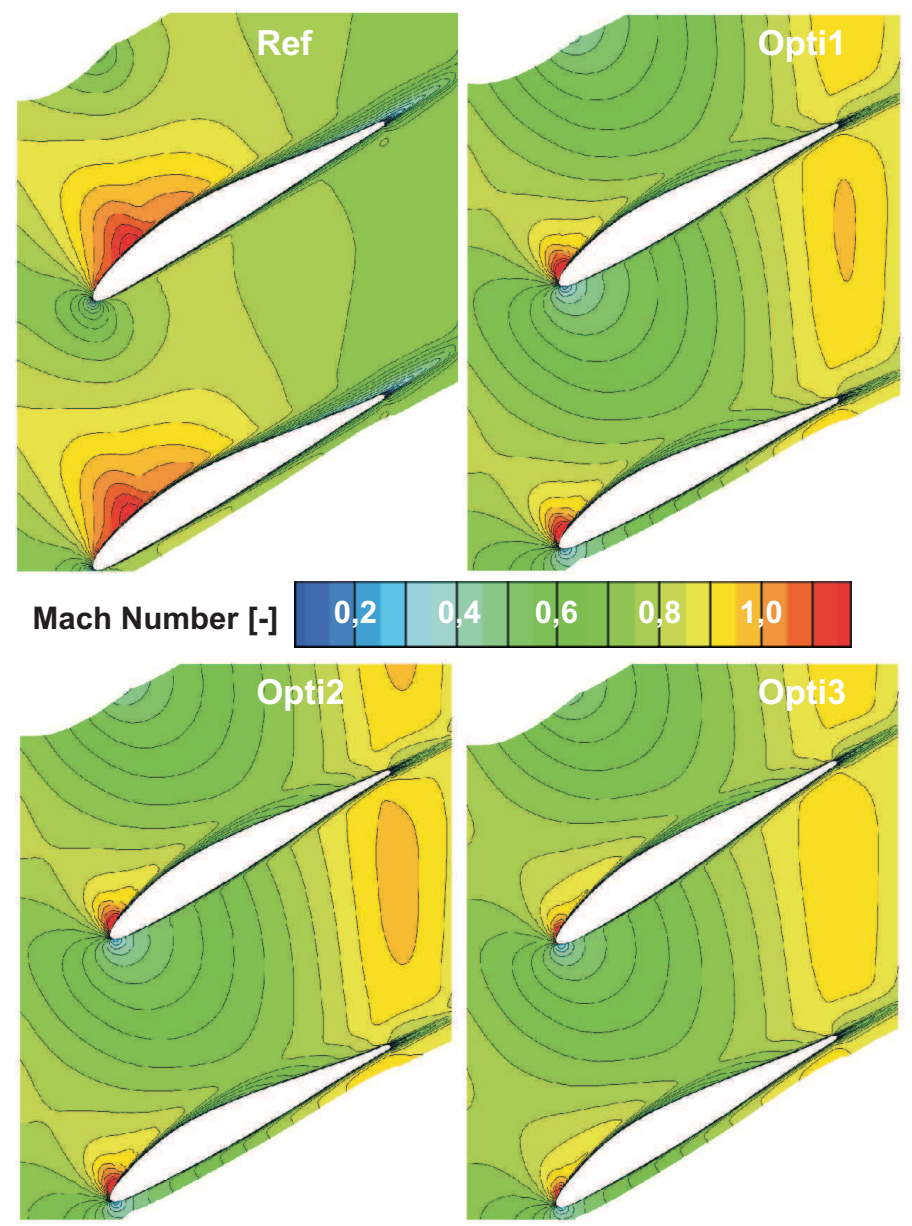

Figure 11. DISTRIBUTION OF MACH NUMBER IN S1 SECTION AT $10 \%$ HEIGHT

the three selected optimal members, For Opti3, it is slightly lifted in the region of the trailing edge, whereas the other two designs display an increased contraction.

In principle the $3 \mathrm{D}$ design of the blades is similar, scaled to the respective flow path. The blades themselves are curved in an s-shape. The leading edges are bent exceedingly in upstream direction in the tip region. Their 3D shapes are shown in Fig. 12 so that the trailing edge is ahead, respectively. In the tip region, the blades additionally are bent to the suction side. The effect is illustrated by the plotted circumferential distributions regarding the magnitude of the vorticity. Looking at the three optimal designs, the tip vortex is lessened significantly in contrast to the original blade design. The magnitude of the vorticities decreases towards the designs featuring a better value for the first objective (efficiency), indicating that the tip vortex also is declined. The distribution of Mach number in S1 section at $90 \%$ height of Opti3 in Fig. 9 also show the tip vortex indicated by their contour lines in the region of the trailing edge. Compared to the initial 

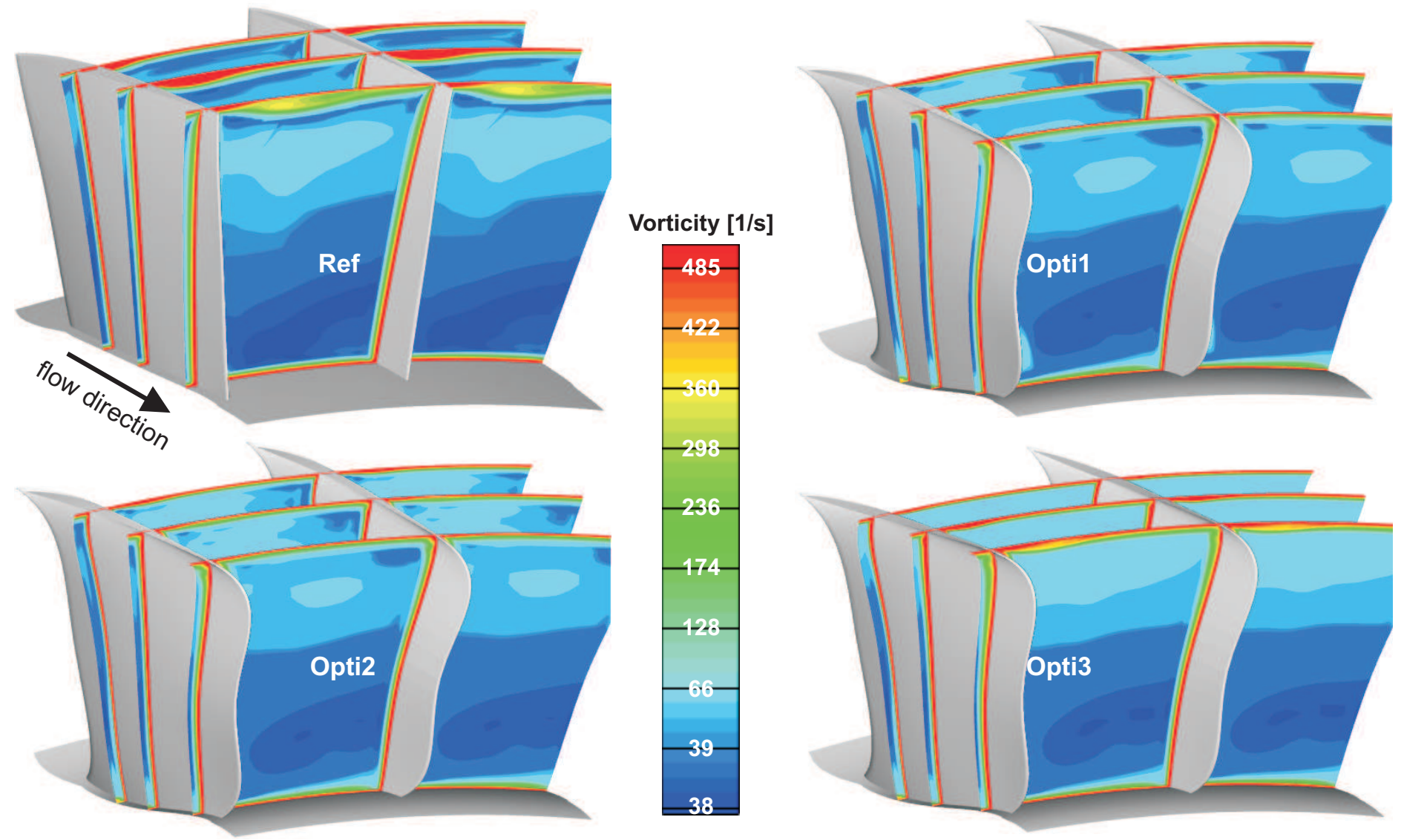

Figure 12. DEVELOPMENT OF THE TIP VORTEX OF THE OPTIMIZED ROTORS AND THE REFERENCE

design it is moved further downstream and closer to the suction side. The higher entropy values at $95 \%$ height for Opti3 in view of Opti1 and Opti2, as seen in Fig. 8, approve the existence of a relatively stronger tip vortex. Additionally, here the deviation rises here versus the initial design.

Generally, the inlet Mach numbers for the three selected optimal designs are lowered, whereupon the outlet Mach numbers are raised, especially in the hub region. The strong contraction of the flow path in the region of rotor 3 corresponds to a reduced aerodynamic load. In Fig. 10, the radial distributions of the deflection, the total temperature ratio, and the total pressure ratio are illustrated, respectively. The increased relative flow angle, caused by the lower inlet Mach number and so the flow path optimization, is mainly responsible for the higher deflection as shown by its radial distribution in Fig. 10a. Just for Opti3 the deflection is lower than for the initial design. The Mach number distribution at $10 \%$ height is depicted in Fig. 11 to display the differing deflection distribution in the hub region in contrast to the initial design. The characteristics emphasize that the absolute level of the Mach number is decreased on the suction side of the profile and the stagnation point is moved to the pressure side. This incidence leads to an improved air flow on the suction side because the maximal velocities are shifted upstream and a smoother exhaust flow is formed reducing the wake. An adaptation of the geometric inlet and outlet blade angles has not been considered in the optimization.

In the matter of Opti1, the raised deflection implies an increase in the total pressure ratio $\pi_{t, R o t}$, whereas the total temperature ratio is slightly raised as shown in Fig. 10. So the energy level of the fluid is heightened without an increase in the necessary load. The isentropic efficiency is improved by $2,6 \%$ to $\eta_{i s, R o t 3}=92,6 \%$. At the same time, the overall efficiency is increased from $\eta_{\text {pol,Ref }}=89,1 \%$ to $\eta_{\text {pol,opti1 }}=90,4 \%$ traded versus a higher load in stator 3 . Hence, the average diffusion factor of stator 3 is raised by $4 \%$ from $D F_{\text {avg,Ref }}=0,5061$ to $D F_{\text {avg, } O p t i 1}=0,5272$. The overall total pressure ratio is increased by the higher pressure increase in rotor 3 to $\pi_{t, \text { Opti1 }}=2,052$ in contrast to the original design $\pi_{t, R e f}=2,017$.

The design Opti2 describes a compromise between the two objectives. The characteristics of Opti1 and Opti2 are qualitatively related as illustrated in Fig. 10. The total pressure ratio is barely below the reference and the rotor here is slightly un- 
loaded. The efficiency of the rotor and the overall efficiency reach $\eta_{i s, \text { Rot } 3}=92,0 \%$ and $\eta_{\text {pol }, \text { Opti } 1}=90,4 \%$, respectively. The magnitude of the diffusion factors of stator 3 is kept on the level of the reference.

The average level of the diffusion factors in stator 3 drops by $8 \%$ for the design Opti3. Figure 10 shows that the load of the rotor is shifted to the tip region looking at the radial distribution of the total pressure ratio and the total temperature ratio. As already mentioned, the modulation of the flow is not optimal in the tip region for the efficiency of rotor 3. Thus, the following stator is unloaded. The overall efficiency and the efficiency of rotor 3 are still raised by $0,7 \%$ and $2,0 \%$ respectively.

\section{CONCLUSION}

In this paper, a linked CFD-solver has been introduced successfully combining the 3D simulation with a throughflow solver. The complex exchange of the flow information is implemented, taking into account certain limitations for the throughflow solver. Although the linking is performed iteratively, the linked solver converges just as fast as a sole 3D-RANS calculation. Therefore, the linked solver features new options configuring the fitness functions without a decisive delay.

The potential of the linked CFD-solver has been demonstrated in connection with the multi-objective optimization at IDAC3. The enclosed example illustrates the optimization for one blade row to be optimized and one operating point. Looking at the Pareto optimals, the improvements of the third rotor are leading to a better performance of the entire axial compressor. The throughflow solver enables the assessment of the influence on the adjoining blade rows. Thus, the additional options expand the possible field of applications for the optimization by using objectives beyond the 3D optimized blades.

\section{ACKNOWLEDGEMENTS}

This work was supported by Arbeitsgemeinschaft Hochtemperatur Gasturbine (AG Turbo), Germany, and the German Federal Ministry of Economics and Technology (BMWi), Förderkennzeichen 0327720A.

\section{REFERENCES}

[1] Ahmed, R., and Lawerenz, M., 2003. "On the AeroMechanical Design of Multistage Axial Compressors using Parallel Optimization Algorithms". 16'th Symposium on air breathing engines, Number ISABE 2003-17.

[2] Rai, M. M., 2006. "Single- and Multiple-Objective Optimization with Differential Evolution and Neural Networks". VKI Lecture Series 2006-03: Introduction to Optimization and Multidisciplianry Design.
[3] Giannakoglou, K. C., and Karakasis, M. K., 2006. "Hierarchical and Distributed Metamodel-Assisted Evolutionary Algorithms". VKI Lecture Series 2006-03: Introduction to Optimization and Multidisciplianry Design.

[4] Chung, H.-S., and Alonso, J. J., 2004. "Multiobjective Optimization Using Approximation Model-Based Genetic Algorithms". 10th AIAA/ISSMO Symposium on Multidisciplinary Analysis and Optimization, AIAA 2004-4325.

[5] Voß, C., Aulich, M., Kaplan, B., and Nicke, E., 2006. "Automated Multiobjective Optimisation in Axial Compressor Blade Design". ASME Paper GT2006-90420.

[6] Fay, G., 2002. "Zur Berechnung der Meridianströmung subsonischer Axialverdichter auf der Basis der umfangsgemittelten Navier-Stokes-Gleichungen". Dissertation, Fakultät für Maschinenbau der Universität Kassel.

[7] Kügeler, E., Weber, A., and Lisiewicz, S., 2001. "Combination of a Transition Model with a Two-Equation Turbulence Model and Comparison with Experimental Results". Proceedings of the 4th European Turbomachinery Conference, Florence, Italy, ATI-CST-076/01.

[8] Nürnberger, D., 2004. "Implizite Zeitintegration für die Simulation von Turbomaschinenströmungen". Dissertation, Fakultät für Maschinenbau der Ruhr-Universität Bochum.

[9] Çetin, M., Üçer, A. c., Hirsch, C., and Serovy, G. K., 1987. "Application of Modified Loss and Deviation Correlations to Transonic Axial Compressors". AGARD-R745.

[10] Johnsen, I. A., and Bulloc, R. O., 1965. "Aerodynamics Design of Axial-Flow Compressors". NASA SP-36.

[11] Abbas, H. A., Sarker, R., and Newton, C., 2001. "PDE: A Pareto-Frontier Differential Evolution Approach for MultiObjective Optimization Problems". Proceedings of the Congress on Evolutionary Computation, 2, May, pp. 971978.

[12] Dorfner, C., Nicke, E., and Voss, C., 2007. "AxisAsymmetric Profiled Endwall Design Using Multiobjective Optimization Linked with 3D-RANS-Flow-Simulations". ASME Paper GT2007-27268.

[13] Piegl, L., and Tiller, W., 1997. "The Nurbs Book, Monographs in Visual Communication". 2nd ed., ISBN 3-54061545-8, Springer Verlag.

[14] Weber, A., 2004. "3D Structured Grids for Multistage Turbomachinery Applications based on G3DMESH". 1st revision ed. Institute of Propulsion Technology, German Aerospace Centre, Cologne, February, DLR IB-325-05-04.

[15] Bohne, A., 2002. "Einfluss von instationärer Strömung und Turbulenz auf die Profilumströmung und das Grenzschichtverhalten moderner Beschaufelungen mehrstufiger Axialverdichter im off-design-Bereich". Forschungsvereinigung Verbrennungskraftmaschinen e. V., Frankfurt, Abschlussbericht Vorhaben 694.

[16] Hoynacki, A., 1999. "Einfluss von instationärer Strömung 
und Turbulenz auf die Grenzschicht und auf die Druckverteilungen von Beschaufelungen moderner mehrstufiger Verdichter". Forschungsvereinigung Verbrennungskraftmaschinen e. V., Frankfurt, Abschlussbericht Vorhaben Nr. 601.

[17] Hoynacki, A., Gallus, H., and Niehuis, R., 1999. "Unsteady Flow Phenomena in a Three Stage Axial Compressor with Controlled Diffusion Airfoils". IGTC'99 Kobe, 1999-1022. 